J. Phys. IV France 127 (2005) 105-109

(C) EDP Sciences, Les Ulis

DOI: $10.1051 /$ jp4:2005127016

\title{
Détection d'activations corticales par des méthodes d'optique diffuse résolues en temps
}

\author{
B. Montcel, R. Chabrier, M. Torregrossa et P. Poulet \\ Institut de Physique Biologique, UMR ULP/CNRS 7004, 4 rue Kirschleger, \\ 67085 Strasbourg, France
}

\begin{abstract}
Résumé. Nous décrivons un appareil pour l'imagerie proche infrarouge (PIR) en neurologie basé sur des diodes laser picosecondes, un tube photomultiplicateur à galette de micro-canaux (MCP-PMT) à huit anodes, et des cartes de comptage de photons résolus en temps (TCSPC). La méthode des éléments finis (MEF) est utilisée pour résoudre l'équation de diffusion des photons sur des modèles du crâne obtenus à partir d'images de résonance magnétique (IRM). Ces simulations suggèrent que les techniques résolues en temps apportent des informations sur la profondeur des variations des propriétés optiques, et permettent d'améliorer la détection d'activations corticales. Les premiers résultats expérimentaux obtenus sur le cortex moteur confirment ces hypothèses.
\end{abstract}

\section{INTRODUCTION}

Il a été démontré précédemment que les variations locales de la perfusion cérébrale et de la saturation en oxygène de l'hémoglobine, induites par une activation corticale, peuvent être mesurées à travers le crâne grâce à la spectroscopie PIR [1]. Dans la perspective d'obtenir une image de ces paramètres, les techniques résolues en temps sont prometteuses car elles récupèrent plus d'information que n'importe quelle autre méthode, elles devraient ainsi être les plus à même de fournir des images fonctionnelles. Dans ce contexte, l'utilisation d'un MCP-PMT et de modules TCSPC semble être pertinente.

Un des inconvénients de la spectroscopie PIR continue est qu'une mesure, pour une configuration spécifique de la source et du détecteur, ne permet pas de différencier une variation superficielle d'une variation profonde des paramètres optiques. Les techniques résolues en temps peuvent sans doute participer à la résolution de ce problème, à condition de savoir quelles zones de la tête sont analysées par cette configuration de la source et du détecteur. A cet effet la propagation lumineuse et les cartes de sensibilité sont simulées à partir de l'équation de diffusion en deux dimensions résolue grâce à la MEF. La cartographie des propriétés optiques de la tête a été obtenue à partir de la segmentation d'IRM.

Cet article présente une méthode de simulation permettant d'étudier l'amélioration apportée par les techniques optiques résolues en temps, concernant la profondeur des variations des paramètres optiques et la détection des activations corticales. Nous décrivons le dispositif expérimental ainsi que ses spécifications. Enfin nous discutons de l'adéquation des simulations avec les résultats expérimentaux obtenus sur le cortex moteur, et des paramètres fonctionnels accessibles grâce à cette instrumentation.

\section{SIMULATION}

Le modèle utilisé est basé sur la segmentation d'une coupe coronale d'IRM de la tête. Cette image a été segmentée manuellement en cinq types de tissus, la peau, l'os du crâne, le liquide céphalo-rachidien (LCR), la matière grise (MG) et la matière blanche $(\mathrm{MB})$. Leurs propriétés optiques à la longueur d'onde de $800 \mathrm{~nm}$ apparaissent dans le tableau 1. Ces propriétés tiennent compte de la diffusion optique dans l'espace subarachnoïdien qui contient le LCR [2]. Nous considérons que l'approximation de diffusion est valide dans ce modèle. L'équation de diffusion est résolue à l'aide d'une MEF en 2 dimensions [3], ce qui permet de simuler la propagation lumineuse et d'obtenir les profils de l'intensité entrant dans le détecteur en fonction du temps, les TPSF. (Temporal Point Spread Function) Le maillage nécessaire à la MEF est obtenu grâce à partir de l'image IRM précédemment segmentée. 
Tableau 1. Propriétés optiques des tissus de la tête à $800 \mathrm{~nm}$.

\begin{tabular}{llllll}
\hline Types de tissus & Peau & Os & LCR & MG & MB \\
\hline Coefficient de diffusion réduite $\mu_{\mathrm{s}}{ }_{\mathrm{s}}\left(\mathrm{mm}^{-1}\right)$ & 1,9 & 1,6 & 0,25 & 2,2 & 9,1 \\
Coefficient d'absorption $\mu_{\mathrm{a}}\left(\mathrm{mm}^{-1}\right)$ & 0,018 & 0,016 & 0,004 & 0,036 & 0,014 \\
\hline
\end{tabular}

\subsection{Cartes de sensibilité}

Les cartes de sensibilité représentent l'influence de la variation des propriétés optiques sur le signal détecté. La méthode de calcul de ces cartes a été décrite en détail dans une autre étude [4]. La Figure 1 représente les cartes de sensibilité en absorption pour quatre distances différentes entre la source et le détecteur. L'influence du faible coefficient de diffusion du LCR apparaît sur ces cartes. La région de haute sensibilité, représentée par le contour le plus intérieur, pénètre dans le cerveau mais est confinée dans la matière grise. Il est impératif de placer le détecteur à une distance suffisamment éloignée de la source pour pouvoir détecter une variation corticale. Les contraintes expérimentales sont très variables d'un sujet à l'autre, ainsi pour les expériences in vivo présentées dans la suite de l'article nous avons placé le détecteur à $30 \mathrm{~mm}$ de la source.

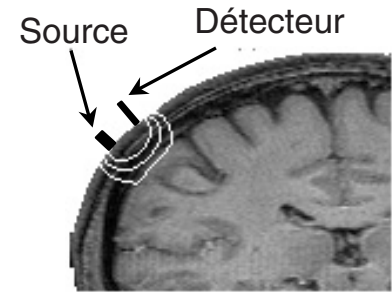

$15 \mathrm{~mm}$

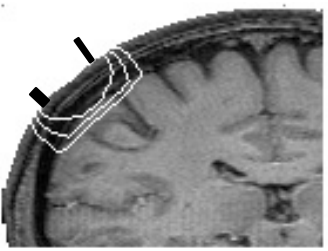

$30 \mathrm{~mm}$

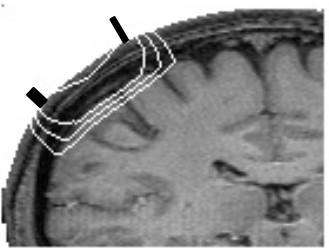

$45 \mathrm{~mm}$

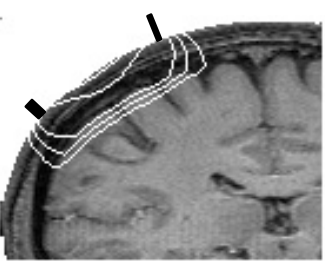

$60 \mathrm{~mm}$

Figure 1. Cartes de sensibilité en $\mu_{\mathrm{a}}$ pour quatre distances différentes entre la source et le détecteur. (Intégration le long de toute la TPSF) Les contours blancs représentent les lieux ou la sensibilité est de $10 \%$, $1 \%$ et $1 \%$ du maximum de chaque image.

Il est aussi intéressant de considérer les cartes de sensibilité en fonction du temps d'arrivée des photons au lieu d'intégrer sur tout le signal temporel. La Figure 2 présente les cartes de sensibilité pour des photons arrivés précocement et pour des photons plus tardifs.

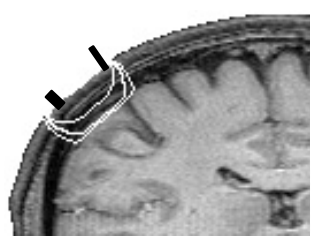

$0,8 \mathrm{~ns}$

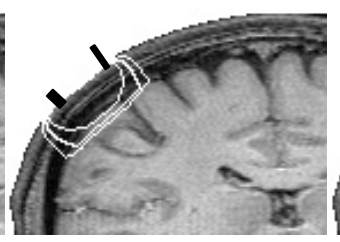

$1,6 \mathrm{~ns}$

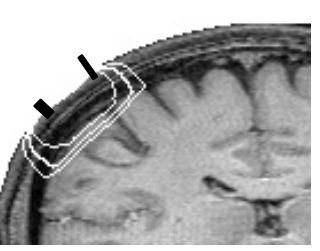

$2,4 \mathrm{~ns}$

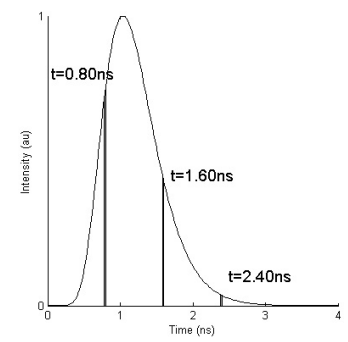

Figure 2. Cartes de sensibilité en $\mu_{\mathrm{a}}$ à trois instants différents de la TPSF pour une distance entre la source et le détecteur de 30mm et TPSF correspondant à cette géométrie. Les contours blancs représentent les lieux ou la sensibilité est de $10 \%, 1 \%$ et $1 \%$ o du maximum de chaque image.

Nous remarquons que les photons tardifs, ayant suivi un chemin optique plus long, ont pénétré plus profondément dans le cerveau. Ceci laisse présager une amélioration de la détection des activations corticales obtenue en ne considérant que les photons les plus tardifs, ainsi que la possibilité d'obtenir une information sur la localisation en profondeur de la variation de perfusion. 


\subsection{Simulation d'activations cérébrales}

Nous avons simulé une activation du cortex moteur en augmentant le coefficient d'absorption d'une région activée représentée sur la Figure 3. Cette région, située dans la matière grise, a une longueur d'environ $11 \mathrm{~mm}$ et une profondeur de $8 \mathrm{~mm}$. Nous avons aussi simulé une augmentation superficielle de la perfusion en augmentant le coefficient d'absorption de la peau. Ceci peut être reproduit expérimentalement lors d'une manœuvre de Valsalva.

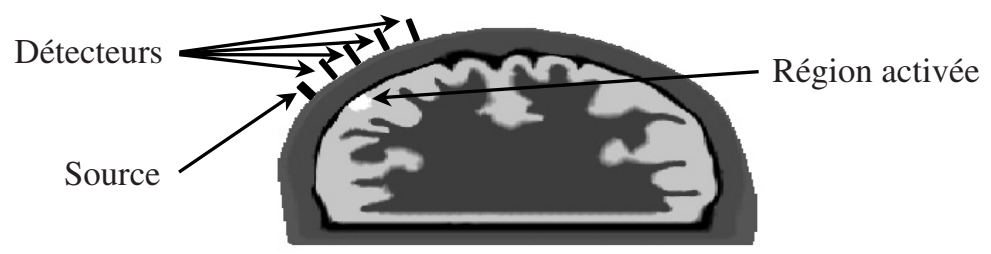

Figure 3. Modèle d'activation corticale. Le coefficient d'absorption est représenté en échelle de gris inversée.

\section{CONDITIONS EXPÉRIMENTALES}

\subsection{Instrumentation}

L'instrumentation, décrite en détail dans un article précédent [5], est composée d'un système multicanaux de diodes laser picosecondes (Sepia, Picoquant) et d'un MCP-PMT à huit anodes (R4110U, Hamamatsu) suivi de huit cartes TCSPC (SPC 134, Becker \& Hickl), comme décrit sur la Figure 4. Les têtes laser $(690,785$ et $830 \mathrm{~nm})$ sont déclenchées séquentiellement à une fréquence de répétition de 5 à $80 \mathrm{MHz}$. La largeur à mi-hauteur de l'impulsion est inférieure à 70ps pour une puissance moyenne inférieure à $1 \mathrm{~mW}$ à $80 \mathrm{MHz}$. Les impulsions laser sont conduites par des fibres optiques multimode. La lumière émergeant des tissus est captée par huit fibres optiques multimodes ayant un cœur de $1 \mathrm{~mm}$ de diamètre (Ocean Optics). Ces fibres sont connectées à un faisceau à huit canaux. La largeur à mi-hauteur de la réponse impulsionnelle de l'ensemble de l'instrumentation est d'environ 200ps. Les fibres optiques, le MCP-PMT et l'électronique sont les sources principales de cet élargissement temporel. La stabilité du signal mesuré est d'environ $1 \%$.

Un casque en thermoplastique (Aquaplast), qui apparaît sur la Figure 4, a été moulé sur la tête du sujet. Un autre capteur, également utilisé, a été adapté à partir d'un système EEG largement utilisé en milieu clinique.

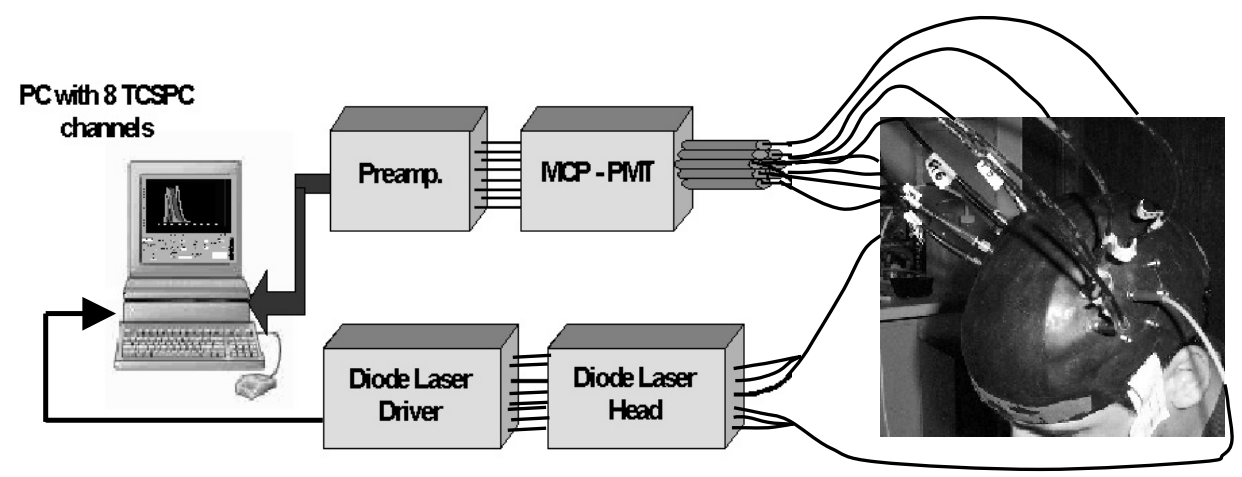

Figure 4. Schéma du montage expérimental d'activation du cortex moteur. 


\subsection{Paradigmes expérimentaux}

La stimulation du cortex moteur a été réalisée grâce à des mouvements rapides des doigts. Cinq cycles consistant en 32s de repos, suivies de 32s de stimulation puis de 32s de repos étaient enchaînés puis accumulés. La manœuvre de Valsalva consiste à expirer en se bouchant le nez et la bouche, ce qui induit une augmentation de la pression intra-thoracique et entraîne une hausse de la perfusion superficielle, notamment au niveau du crâne. Quatre cycles consistant en 32s de repos suivies de 15 s de stimulation puis de 49s de repos étaient enchaînés puis accumulés. Dans les deux cas les TPSF à 830nm ont été mesurées à une fréquence d'acquisition de $1 \mathrm{~Hz}$.

\section{VALIDATION EXPÉRIMENTALE}

Les résultats sont présentés dans le but de répondre aux deux questions suivantes. Pouvons nous différencier une activation du cortex moteur d'une manœuvre de Valsalva, en terme de profondeur de la variation de perfusion? Pouvons nous améliorer la détection d'une activation corticale en ne considérant qu'une partie de la TPSF?

\subsection{Variation corticale ou superficielle?}

Nous avons évalué la différence point à point entre la TPSF durant l'activation et la TPSF au repos. La Figure 5 présente cette différence superposée à la TPSF simulée au repos.
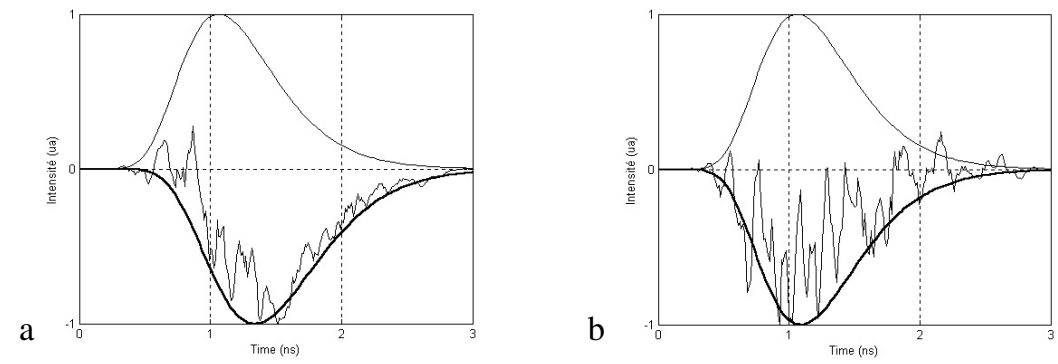

Figure 5. Différence point à point entre la TPSF durant l'activation et celle au repos. TPSF au repos (— de 0 à 1$)$, différence simulée (—de -1 à 0 ) et différence expérimentale ( - de -1 à 0$)$, mesures à $30 \mathrm{~mm}$ et à $830 \mathrm{~nm}$. Toutes les courbes sont normées à 1. a: Activation corticale. b: Manœuvre de Valsalva.

La simulation de la différence dans les cas de l'activation corticale atteint son maximum dans le front descendant de la TPSF, alors que dans le cas de la variation superficielle, les maxima de la TPSF et de la différence sont confondus. Ce comportement caractéristique se retrouve dans les courbes expérimentales, ce qui atteste du caractère superficiel ou profond de la variation.

\subsection{Activation corticale}

Nous avons comparé notre système résolu en temps avec un système continu en mesurant le rapport contraste à bruit (RCB) sur le signal de toute la TPSF (équivalent à système continu) et sur 8 zones de 300ps de largeur le long de la TPSF. Le RCB a été évalué en calculant le ratio entre la variation du signal due à l'activation (contraste) et la déviation standard du signal au cours de la mesure (bruit). Le contraste augmente avec le temps d'arrivée des photons, mais le RCB atteint un maximum pour la zone 6 (Figure 6). Ceci s'explique par l'augmentation du bruit due au faible taux de comptage pour les photons tardifs. L'amélioration du CNR dans la zone optimale par rapport au système continu est d'environ $24 \%$. 


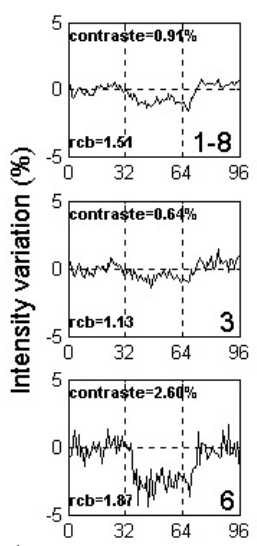

a)
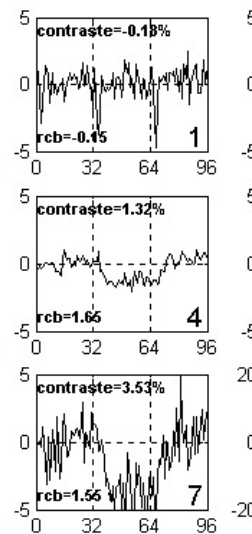

Time (s)
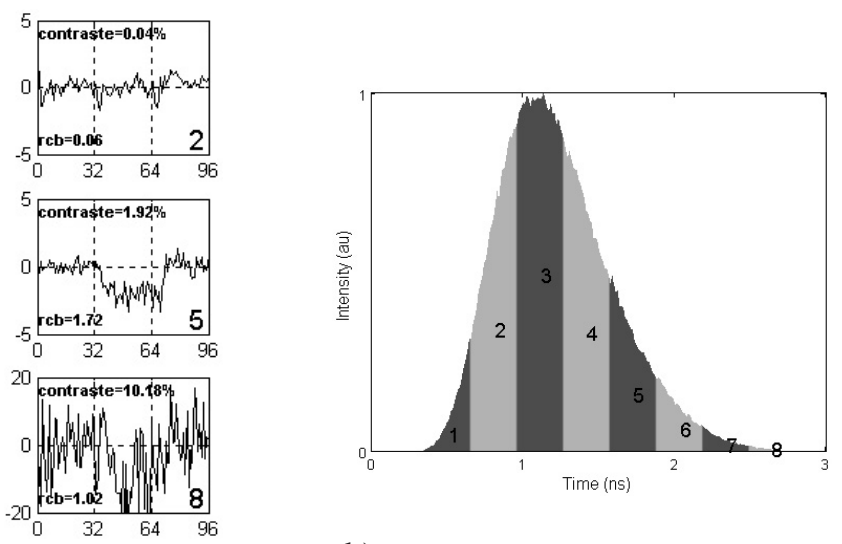

b)

Figure 6. a) Contraste et rapport contraste à bruit (rcb) sur toute la TPSF (graphe 1-8) et sur différentes zones de la TPSF (graphes 1 à 8), mesurée sur le cortex moteur à $30 \mathrm{~mm}$ et à $830 \mathrm{~nm}$. Le graphe 8 a une échelle différente des autres. b) Zones 1 à 8 de la TPSF.

\section{DISCUSSION ET CONCLUSION}

Les premiers résultats expérimentaux confirment que les techniques résolues en temps permettent de différencier des variations superficielles de la perfusion et de réelles activations corticales et qu'il est possible d'améliorer significativement la détection de ces activations cérébrales en ne prenant en compte que les photons tardifs du signal résolu en temps. De plus en utilisant les trois longueurs d'onde à notre disposition et en considérant la loi de Beer-Lambert, il est possible de calculer les variations de concentrations moyennes d'oxy- et de deoxy-hémoglobine dans la zone analysée. Dans ce cas, le chemin optique moyen dans cette zone est évalué grâce à la mesure du temps moyen de propagation dans les tissus. Nous poursuivons l'amélioration du système expérimental en augmentant le nombre de capteurs sur la zone d'intérêt, afin d'obtenir un véritable système d'imagerie fonctionnelle.

\section{Remerciements}

Ces travaux ont bénéficié des soutiens financiers des Hôpitaux Universitaires de Strasbourg, de la région Alsace et du Ministère de la Recherche, action concertée incitative "Technologies pour la santé".

\section{Bibliographie}

[1] A. Villringer, B. Chance "Non-invasive optical spectroscopy and imaging of human brain function", Trends Neurosci. 20, 435-442 (1997).

[2] Y. Fukui, Y. Ajichi, E. Okada "Monte Carlo prediction of near-infrared light propagation in realistic adult and neonatal head models", Applied Optics 42, 2881-2887 (2003).

[3] S. R. Arridge, M. Schweiger, M. Hiraoka and D. T. Delpy "A finite element approach for modelling photon transport in tissue”, Med. Phys. 20, 299-309 (1993).

[4] S. R. Arridge and M. Schweiger "Photon-measurement density functions. Part2: Finite-elementmethod calculations", Applied Optics 34, 8026-8037 (1995).

[5] P. Poulet, C.V. Zint, M. Torregrossa, W. Uhring and B. Cunin "Comparison of two time-resolved detectors for diffuse optical tomography: photomultiplier tube - time-correlated single photon counting and multi-channel streak camera", Optical Tomography and Spectroscopy of Tissue V, Britton Chance, Robert Alfano, Bruce Tromberg, Mamoru Tamura, Eva Sevick-Muraca, eds., Proc. SPIE, 4955, 154-163 (2003). 